\title{
Target weight gain for moderately wasted children during supplementation interventions - a population-based approach
}

\author{
André Briend ${ }^{1, *}$, Jan Van den Broeck ${ }^{2}$ and Lars T Fadnes ${ }^{2}$ \\ 'Department of International Health, University of Tampere Medical School, FIN-33014, Tampere, Finland: \\ ${ }^{2}$ Centre for International Health, University of Bergen, Norway
}

Submitted 25 February 2011: Accepted 8 June 2011: First published online 11 August 2011

\begin{abstract}
Objective: In malnourished populations, the weight-for-height $Z$-score (WHZ) distribution is shifted to the left. The aim of nutrition interventions should be to restore a normal WHZ distribution for the whole population. The present paper examines the WHZ change needed by each individual to achieve this objective. Design: We developed a mathematical model of required individual change in WHZ as a function of characteristics of the initial population to restore a normal distribution. This model was then tested by simulating WHZ change needed to restore a normal WHZ distribution in a test population.

Setting: A rural area of Democratic Republic of the Congo with a high prevalence of undernutrition.

Subjects: Children under 5 years of age.

Results: To restore a normal distribution for the whole population, the WHZ of all children should be shifted. The desired WHZ change of each individual should be higher when the individual's initial WHZ is low, when the mean WHZ of the whole population is low and, for the most wasted individual, when the variance of WHZ and WHZ change in the population are high. Using the suggested model in a simulation on the test population resulted in a WHZ distribution close to the growth standard.

Conclusions: To restore a normal WHZ distribution in wasted populations, nutritional programmes should cover the whole population with a higher weight gain in areas where mean WHZ is low.
\end{abstract}

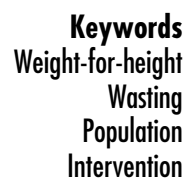

Most programmes addressing moderate wasting with a weight-for-height $Z$-score (WHZ) between -3 and -2 discharge children when they reach a fixed threshold ${ }^{(1)}$. This threshold is often set at a WHZ above -2 or -1 . These fixed cut-offs have limitations. Reaching a WHZ of -2 may be too low for some children, who may initially have had a WHZ in the upper normal range and have entered the nutritional supplementation programme when they fell just below -2 . These children might reach this discharge criterion with a small weight gain while still being substantially under their initial physiological weight. On the other hand, the -1 cut-off is not a good option either, as about $16 \%$ of the children in a normal well-nourished population are below this cut-off. Problems associated with the definition of wasting based on a fixed cut-off are also suggested by an observed mismatch between the clinical aspect of the child and his/her anthropometric status ${ }^{(2)}$.

Another limitation of the current approach is that it assumes that only children below the arbitrary $-2 Z$-score cut-off are underweight and will benefit from nutritional supplementation. This is unlikely to be true. In a context of malnutrition, the whole WHZ distribution is usually shifted to the left, with a limited change in the variance of the WHZ distribution, indicating that all children are below their optimum WHZ ${ }^{(3-5)}$. This situation indicates a sick population rather than only sick individuals, as described in another context ${ }^{(6)}$.

Ideally, nutritional interventions should have the objective to restore the normal WHZ distribution in line with the WHO growth standards (WHZ mean $=0 ; \quad W H Z ~ S D=1$, WHZ variance $=1$ ) which represents how a population of well-nourished children is supposed to grow ${ }^{(7)}$. A population with a left-shifted WHZ distribution and an unchanged variance could be shifted back to a distribution like the growth standard with an intervention resulting in a uniform WHZ increase for all children across the WHZ distribution. This is unlikely to happen in real life, as WHZ increases during interventions have a great variability. For instance, in a recent supplementation study in moderately wasted children in Niger, the standard deviation of weight gain was comparable to weight gain itself ${ }^{(8)}$. 
The objective of the present study was to estimate the plausible WHZ change that each individual in a wasted population will need to restore the normal WHZ distribution in line with the WHO growth standards representing a well-nourished population.

\section{Methods}

The present paper first develops a mathematical model to estimate the individual's WHZ change that is needed during nutritional interventions to shift the WHZ distribution to the right to a standard deviation of 1 and a mean of 0 . Further, a simulation is used to test the validity of this model.

\section{Matbematical model}

The average WHZ change needed to shift the whole distribution of WHZ to the right to get a new distribution with a mean of 0 and a standard deviation of 1 can be estimated using the relationship between the variance of a sum and the variance of each of its terms ${ }^{(9)}$. The details of the calculation are given in the Appendix, where it is shown that the aimed WHZ change ( $\Delta$ WHZ) for each child depends on his/her initial WHZ $\left(\mathrm{WHZ}_{\mathrm{i}}\right)$, the mean and variance of the initial WHZ of the whole population before the intervention (Mean $\mathrm{WHZ}_{\mathrm{i}}$; Var $\mathrm{WHZ}_{\mathrm{i}}$ ) and the variance of $\Delta \mathrm{WHZ}$ (Var $\Delta \mathrm{WHZ}$ ). These variables are linked by the relationships:

$$
f(x)=a+b x
$$

and

$$
\Delta \mathrm{WHZ}=a+b \cdot \mathrm{WHZ}_{\mathrm{i}}
$$

with

$$
b=\left(1-\operatorname{Var} \mathrm{WHZ}_{\mathrm{i}}-\operatorname{Var} \Delta \mathrm{WHZ}\right) /\left(2 \cdot \operatorname{Var} \mathrm{WHZ}_{\mathrm{i}}\right)
$$

and

$$
a=- \text { Mean } \mathrm{WHZ}_{\mathrm{i}}-b \cdot \text { Mean } \mathrm{WHZ}_{\mathrm{i}}
$$

\footnotetext{
Simulation

The model above suggests that the average WHZ change needed to shift the distribution will depend of the variance of $\Delta \mathrm{WHZ}$. To estimate this variance, data from a longitudinal growth study of children from Democratic Republic of the Congo (DR Congo) were examined. That study took place in 1989-1991 and is described in detail elsewhere $^{(10)}$. In brief, a dynamic population including a total of 5652 children under the age of 5 years (average per visit: 4698) was followed for about 2 years. Children were seen six times at 3-month intervals, with weight and height measurements taken each time. Weight was measured undressed with spring scales to the nearest $100 \mathrm{~g}$, and length or height to the nearest millimetre with a locally produced measuring board. No nutritional intervention took place in the area during the study. The study was done in a rural area with a seasonal variation in food availability and nutritional status.
}

The weight and height of each child were used to calculate his/her WHZ based on the WHO growth standards ${ }^{(11)}$. The variance of the change in WHZ (Var $\Delta$ WHZ) was calculated for all 3-month intervals, for which the initial and final WHZ were known.

To test the validity of the model on the observed data, the model was used to estimate the shift in WHZ that was needed to restore the WHZ distribution of the WHO growth standard from the baseline level in the cohort of children from DR Congo during the least food-secure season. The survey visit which had the lowest mean WHZ was used as a reference for this simulation.

To restore the mean of the WHO growth standard, an average $\Delta \mathrm{WHZ}$ was added to the initial WHZ of each child. This added $\Delta$ WHZ was estimated using the initial WHZ of each child using the regression model described above.

To achieve the desired $\Delta \mathrm{WHZ}$ variance, a random number was added to $\Delta \mathrm{WHZ}$ for each child. The average of this added random number was 0 , and thus this did not change the overall mean $\Delta \mathrm{WHZ}$. Its variance was the difference between the desired final variance (variance $=1$ ) and the variance of change in WHZ observed after the addition of the $\triangle \mathrm{WHZ}$ due to regression and before the addition of the random number. The resulting total variance of $\Delta \mathrm{WHZ}$ was the desired variance.

\section{Results}

\section{Estimation of variance of WHZ change}

Among all the 3-months intervals, 3953 had an initial and a final WHZ which could be used to calculate the observed variance of $\Delta \mathrm{WHZ}$ between visits. The variance for $\Delta \mathrm{WHZ}$ for all these intervals was $0 \cdot 63$. This observed variance of $\Delta \mathrm{WHZ}$ was used for the simulation.

\section{Simulation of population shift}

The mean WHZ in the baseline visit in the study population was -0.33 (variance $1 \cdot 02$ ). The change in WHZ that was needed to shift the WHZ distribution to a normal distribution with a mean of 0 and standard deviation of 1 was estimated with the model reproduced in the Appendix, assuming a variance of WHZ change of 0.63 .

To restore the mean WHZ of 0 as expected in a wellnourished population, each child needed to increase his/her weight with an amount given by the regression model from his/her baseline WHZ as described in the Methods section and the Appendix. The WHZ change resulting from this WHZ increase due to regression had a variance of $0 \cdot 10$. To achieve the desired WHZ change variance, a random component with a mean of 0 and a variance of 0.53 was added. The resulting distribution is shown in Fig. 1. Mean $Z$-score after simulation was $-0 \cdot 01$ (SD $1 \cdot 02$ ). The regression explained only a small fraction of WHZ change with an $R^{2}$ of $0 \cdot 14$, and simulated WHZ changes were highly variable even for the same initial WHZ (Fig. 2). 


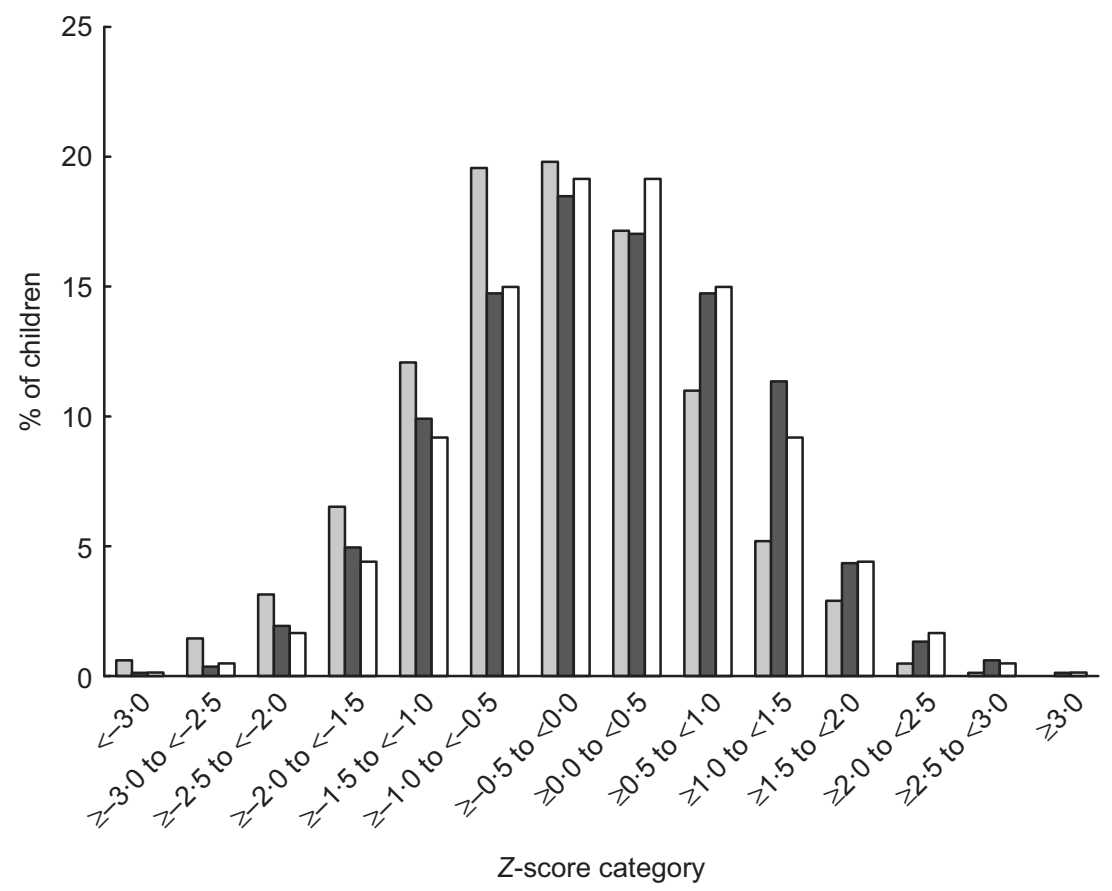

Fig. 1 Distribution shift of weight-for-height $Z$-score $(\mathrm{WHZ})$ after addition of $\Delta \mathrm{WHZ}$ with a random component according to the model: $\square, W H Z_{i}$ (initial $W H Z$ distribution); $\mathbf{a}, W_{H} Z_{f}$ (final $W H Z$ distribution after addition of a $W H Z$ change with a random component); $\square$, normal distribution

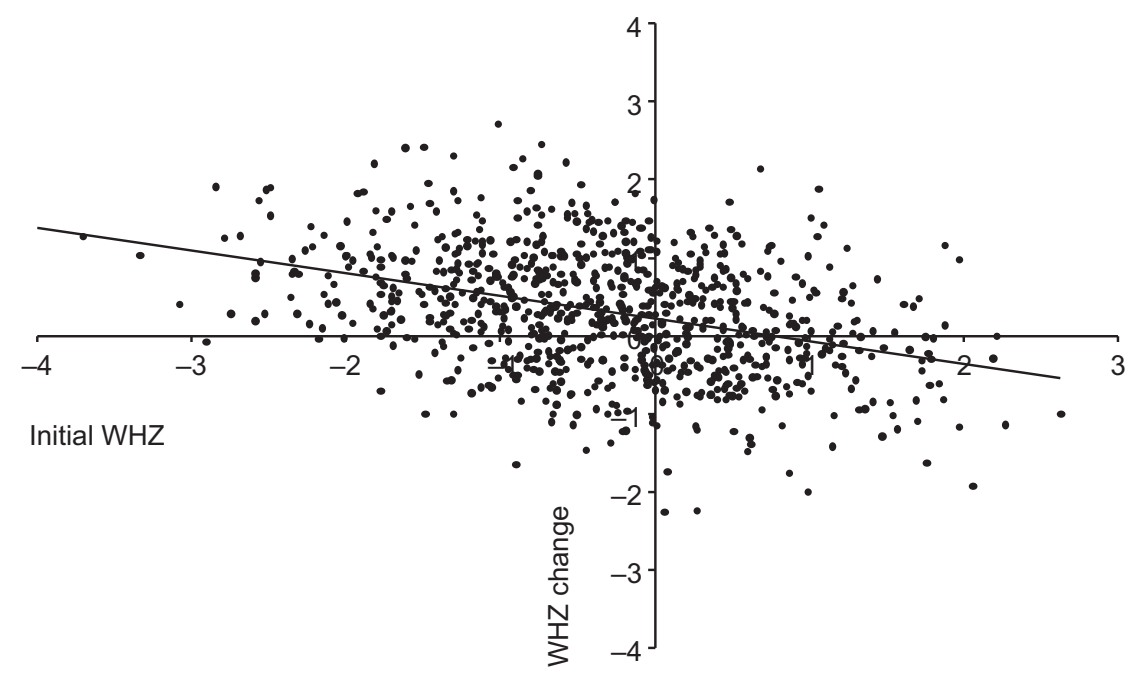

Fig. 2 Change in weight-for-height $Z$-score $(\mathrm{WHZ})$ in relation to initial $\mathrm{WHZ}$ observed in a simulation shifting the WHZ distribution towards a mean of 0 and standard deviation of $1\left(R^{2}=0 \cdot 14\right)$

Even in this simulation which shifted the WHZ distribution to the right, some children lost weight in all WHZ categories. The proportion of children who lost weight among those with an initial WHZ less than -2 was $19 \cdot 5 \%$ (Fig. 3).

\section{Discussion}

Mathematical analysis suggests that to restore a normal WHZ distribution in a population with an initial negative mean WHZ, interventions should in principle aim to change the WHZ of all children. This means that only nutritional programmes that have an effect on the growth of all children can restore a normal WHZ distribution. This is a strong theoretical argument for blanket, community-based nutritional interventions, rather than individual targeted interventions.

The practical implications of these findings should be considered carefully. The health benefits of restoring a normal WHZ distribution are unknown, and they may be small in relation to the costs in settings with a low 


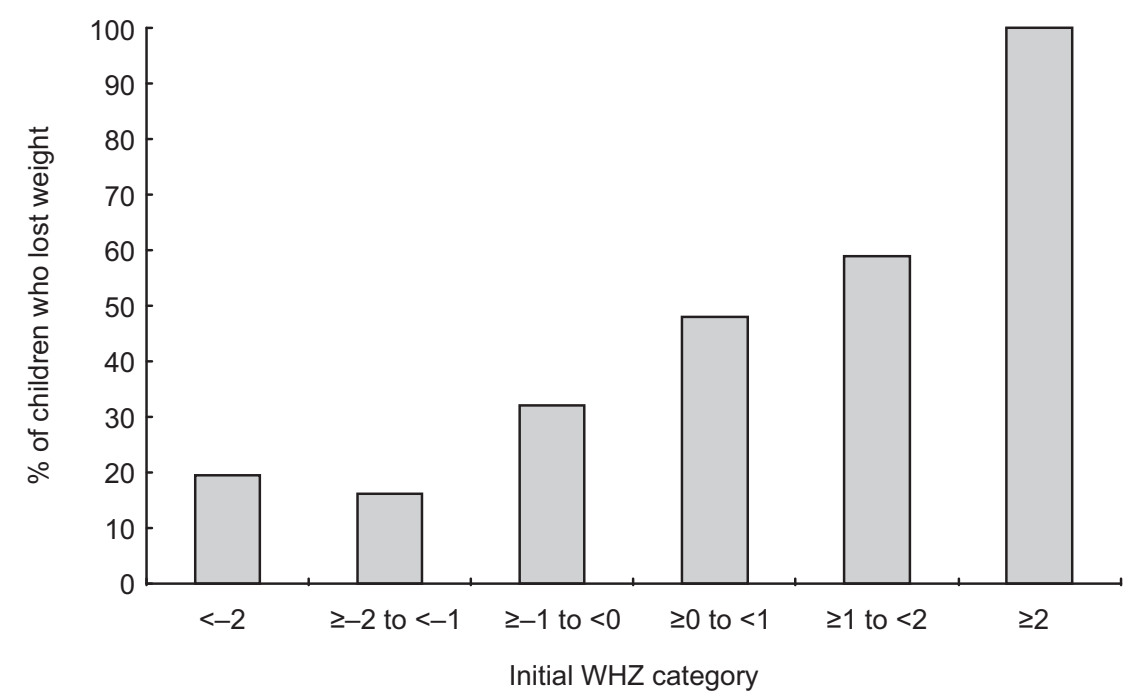

Fig. 3 Percentage of children whose lost weight in different initial weight-for-height Z-score (WHZ) categories in a simulation which shifted the whole $\mathrm{WHZ}$ distribution to the right

prevalence of malnutrition. The relationship between anthropometric deficit and the risk of death is not linear but exponential ${ }^{(12)}$, suggesting that differences in WHZ status on the left part of the distribution are associated with higher number of deaths. In populations with a low prevalence of malnutrition, it may be more advantageous to target nutrition interventions to high-risk groups (i.e. with WHZ less than -2), as these are at an increased risk of mortality ${ }^{(13)}$. This analysis does not provide any information on the risk and benefits on morbidity and mortality of targeted $v$. more universal interventions.

The present study suggests a different response to the same nutritional intervention depending on the initial mean WHZ deficit in the population, the highest WHZ change being required in populations with the lowest mean WHZ. In this respect, the highest weight gains for moderately wasted children have been reported from Niger, an area with a high prevalence of wasting ${ }^{(14)}$. This contrasts with the lower weight gains reported from Malawi where wasting is less common ${ }^{(15)}$. Although part of this difference may be related to different treatment protocols and admission criteria, this is consistent with the hypothesis of a higher weight gain to be expected in areas of high prevalence of wasting.

The model also suggests that the variance of $\mathrm{WHZ}$ in the whole population influences the needed WHZ change, an increased variance requiring a higher WHZ change in the left part of the WHZ distribution. It also suggests that the WHZ change to be expected will be influenced by the variance of WHZ change itself, which may also be influenced by ongoing interventions.

For practical purposes, the model suggests that the mean WHZ change of moderately wasted children should always be superior to the left shift of the mean WHZ of the population. The Appendix (section C) shows how to calculate the needed WHZ change in relation to initial individual WHZ using a simple spreadsheet calculation. When interventions are targeted, the average initial WHZ of targeted individuals should be entered in the model to calculate needed average WHZ change. Calculation of this average requires integrating WHZ up to the cut-off chosen for intervention. This has been done in the Appendix (section D) for a normal distribution and $Z$-scores between -2 and -3 which are commonly used in targeted interventions.

Despite its limitations, the present study has implications for the evaluation of programmes targeted to moderately wasted children. For children with moderate wasting (WHZ between -3 and -2 ), the present analysis suggests that the needed WHZ change will depend on the WHZ of each individual child, but also on the mean WHZ of the whole population, and on the variance of the WHZ change in the population. This suggests that the expected WHZ change in wasted children will be context specific, in particular depending on the mean WHZ of the population.

This simulation also suggests that within a WHZ category, and even when an intervention is successful to shift the WHZ distribution, some wasted children may lose weight, especially if variance of WHZ change is high. It is important to keep in mind that it is hard to predict how individual children will respond to interventions, but the anthropometric effect on a group level should still be a good indicator of whether interventions work appropriately.

Our model suggests that the needed WHZ change for each individual is higher when the average WHZ of the whole population is low. This is consistent with the higher predictive value of diagnostic criteria when malnutrition prevalence is high and identification of those malnourished is imperfect ${ }^{(16)}$.

These proposed needed WHZ changes for wasted children are however tentative. Also, the shift towards a normal distribution is not the only criterion to consider 
when estimating the desirable WHZ shift for targeted programmes. In particular, the risk of relapse and the risk of severe acute malnutrition during follow-up should also be considered.

To summarise, the present study shows that nutritional programmes should cover the whole population with a severity-based approach to restore a normal weightfor-height distribution in wasted populations, aiming at a higher a weight gain in areas where the mean WHZ is low.

\section{Acknowledgements}

This study was supported by the University of Tampere, Department of International Health and the Centre for International Health, University of Bergen, Norway. A preliminary version of this paper was presented at the WHO meeting on Management of Moderate Malnutrition held in Geneva (2-4 December 2009). The authors have no conflict of interest. A.B. developed the mathematical model and wrote the first draft. J.V.d.B. provided the data used for simulation. L.T.F. provided statistical advice. All authors took part in the writing of the manuscript.

\section{References}

1. Navarro-Colorado C (2007) A Retrospective Study of Emergency Supplementary Feeding Programmes. http:// www.ennonline.net/pool/files/research/Retrospective_Study_ of_Emergency_Supplementary_Feeding_Programmes_June\% 202007.pdf (accessed July 2011).

2. Van den Broeck J, Meulemans W \& Eeckels R (1994) Nutritional assessment: the problem of clinical-anthropometrical mismatch. Eur J Clin Nutr 48, 60-65.

3. Yip R \& Scanlon K (1994) The burden of malnutrition: a population perspective. J Nutr 124, 10 Suppl., 2043S-2046S.

4. World Health Organization (1993) Physical Status: The Use and Interpretation of Anthropometry. Report of a WHO Expert Committee, p. 209. Geneva: WHO; available at http://whqlibdoc.who.int/trs/WHO_TRS_854.pdf

5. Golden MHN \& Grellety Y (2002) Population Nutritional status during famine. Standardized Monitoring and Assessment of Relief \& Transition (SMART) Workshop, Technical Working Session: July 23-25. http://www.smartindicators. org/program_files/include/uploads/mg_surveywhzdis.doc (accessed July 2011).

6. Rose G (1985) Sick individuals and sick populations. Int J Epidemiol 14, 32-38.

7. Garza C \& de Onis M (2004) Rationale for developing a new international growth reference. Food Nutr Bull 25, 1 Suppl., S5-S14.

8. Nackers F, Broillet F, Oumarou D et al (2010) Effectiveness of ready-to-use therapeutic food compared to a corn/ soy-blend-based pre-mix for the treatment of childhood moderate acute malnutrition in Niger. $J$ Trop Pediatr 56, 407-413.

9. Armitage P \& Berry G (1994) Statistical Methods is Medical Research, 3rd ed. London: Blackwell Science.

10. Van Den Broeck J, Eeckels R \& Vuylsteke J (1993) Influence of nutritional status on child mortality in rural Zaire. Lancet 341, 1491-1495.
11. World Health Organization (2006) WHO Child Growth Standards. Geneva: WHO; available at http://www. who.int/childgrowth/en/

12. Pelletier DL, Frongillo EA Jr, Schroeder DG et al. (1994) A methodology for estimating the contribution of malnutrition to child mortality in developing countries. J Nutr 124, 10 Suppl., 2106S-2122S.

13. Myatt M, Khara T \& Collins S (2006) A review of methods to detect cases of severely malnourished children in the community for their admission into community-based therapeutic care programs. Food Nutr Bull 27, 3 Suppl., S7-S23.

14. Defourny I, Seroux G, Abdelkader I et al. (2007) Management of moderate acute malnutrition with RUTF in Niger. ENN Field Exchange 31, 2-4; available at http://www. ennonline.net/fex/31/fex31.pdf

15. Matilsky DK, Maleta K, Castleman $\mathrm{T}$ et al. (2009) Supplementary feeding with fortified spreads results in higher recovery rates than with a corn/soy blend in moderately wasted children. J Nutr 139, 773-778.

16. Habicht JP (1980) Some characteristics of indicators of nutritional status for use in screening and surveillance. $\mathrm{Am}$ J Clin Nutr 33, 531-535.

\section{Appendix}

Estimation of WHZ needed to shift WHZ distribution to the right to a final mean of $O$ and standard deviation of 1

A. Calculation of average WHZ change (Mean $\Delta \mathrm{WHZ}$ ) for the whole population

To change the mean in a population to 0 , the required change should be equal to the initial difference from 0 :

$$
\text { Mean } \triangle \mathrm{WHZ}=- \text { Mean } \mathrm{WHZ}_{\mathrm{i}}
$$

B. Calculation of average needed weight gain for a given $W H Z$

The variance of a sum can be calculated using the general formula:

$$
\operatorname{var}(y)=\operatorname{var}\left(x_{1}\right)+\operatorname{var}\left(x_{2}\right)+2 \cdot \operatorname{covariance}\left(x_{1}, x_{2}\right)
$$

The variance of WHZ change (Var $\Delta$ WHZ), the variance of initial WHZ (Var $\mathrm{WHZ}_{\mathrm{i}}$ ) and the variance of the final WHZ (Var $\mathrm{WHZ}_{\mathrm{f}}$ ) are related as follows:

$\operatorname{Var} \mathrm{WHZ}_{\mathrm{f}}=\operatorname{Var} \mathrm{WHZ}_{\mathrm{i}}+\operatorname{Var} \Delta \mathrm{WHZ}+2 \cdot \operatorname{Cov}\left(\mathrm{WHZ}_{\mathrm{i}}, \Delta \mathrm{WHZ}\right)$

The SD of $\mathrm{WHZ}_{\mathrm{f}}$ should be 1 and its variance should be $1^{2}=1$. Hence:

$$
1=\operatorname{Var} \mathrm{WHZ}_{\mathrm{i}}+\operatorname{Var} \Delta \mathrm{WHZ}+2 \cdot \operatorname{Cov}\left(\mathrm{WHZ}_{\mathrm{i}}, \Delta \mathrm{WHZ}\right)
$$

and

$$
\operatorname{Cov}\left(\mathrm{WHZ}_{\mathrm{i}}, \Delta \mathrm{WHZ}\right)=\frac{\left(1-\operatorname{Var} \mathrm{WHZ}_{\mathrm{i}}-\operatorname{Var} \Delta \mathrm{WHZ}\right)}{2}
$$

WHZ change in relation to initial WHZ can be calculated by the general equation for a linear regression model:

$$
\Delta \mathrm{WHZ}=a+b \cdot \mathrm{WHZ}_{\mathrm{i}}
$$


with

$$
a=\text { Mean } \Delta \mathrm{WHZ}-b \cdot \text { Mean } \mathrm{WHZ}_{\mathrm{i}}
$$

or

$$
a=- \text { Mean } \mathrm{WHZ}_{\mathrm{i}}-b \cdot \text { Mean } \mathrm{WHZ}_{\mathrm{i}}
$$

and

$$
b=\frac{\operatorname{Cov}\left(\mathrm{WHZ}_{\mathrm{i}}, \Delta \mathrm{WHZ}\right)}{\operatorname{Var} \mathrm{WHZ}_{\mathrm{i}}}
$$

or

$$
b=\frac{\left(1-\operatorname{Var} \mathrm{WHZ}_{\mathrm{i}}-\operatorname{Var} \Delta \mathrm{WHZ}\right)}{2 \cdot \operatorname{Var} \mathrm{WHZ}_{\mathrm{i}}}
$$

Figures A1, A2 and A3 show the relationship between $\mathrm{WHZ}_{\mathrm{i}}$ and needed $\Delta \mathrm{WHZ}$ for different mean $\mathrm{WHZ}_{\mathrm{i}}$ values for the whole population, for different variances of $\Delta \mathrm{WHZ}$ and for different variances of $\mathrm{WHZ}_{\mathrm{i}}$ of the whole population.

C. Calculation of $W H Z$ change needed in relation to the parameters of the initial population and expected WHZ change variance with a standard spreadsheet

(i) Data entry spreadsheet

Population data

Mean WHZ

B5

Variance WHZ

B6

Variance of WHZ change B7

Individual data

Initial WHZ

B11

(ii) Calculations

Slope

Intercept

(iii) Results

Expected WHZ change for individual

This calculation can be made on several columns for different values of initial WHZ for individuals to reproduce plots as in Figs A1 to A3.

D. Average WHZ for children with a WHZ between -2 and -3 in relation to the average $W H Z$ of the population

$$
\begin{aligned}
& \text { Initial mean WHZ of } \\
& \text { the population }
\end{aligned}
$$

$\begin{array}{ll}0 \cdot 0 & -2 \cdot 32 \\ -0 \cdot 1 & -2 \cdot 32 \\ -0 \cdot 2 & -2 \cdot 33 \\ -0 \cdot 3 & -2 \cdot 33 \\ -0 \cdot 4 & -2 \cdot 34 \\ -0 \cdot 5 & -2 \cdot 35 \\ -0 \cdot 6 & -2 \cdot 35 \\ -0 \cdot 7 & -2 \cdot 36 \\ -0 \cdot 8 & -2 \cdot 37 \\ -0 \cdot 9 & -2 \cdot 38 \\ -1 \cdot 0 & -2 \cdot 38\end{array}$

Average WHZ of children between $Z$-score of

$$
\begin{aligned}
& -3 \text { and }-2 \\
& -2 \cdot 32 \\
& -2 \cdot 32 \\
& -2 \cdot 33 \\
& -2 \cdot 33 \\
& -2 \cdot 34 \\
& -2 \cdot 35 \\
& -2 \cdot 35 \\
& -2 \cdot 36 \\
& -2 \cdot 37 \\
& -2 \cdot 38 \\
& -2 \cdot 38
\end{aligned}
$$

These values were calculated assuming a variance of 1 for the WHZ distribution in the population. These values should be used in the model above (cell B11) to calculate the average WHZ change needed for children between $Z$-scores of -2 and -3 .

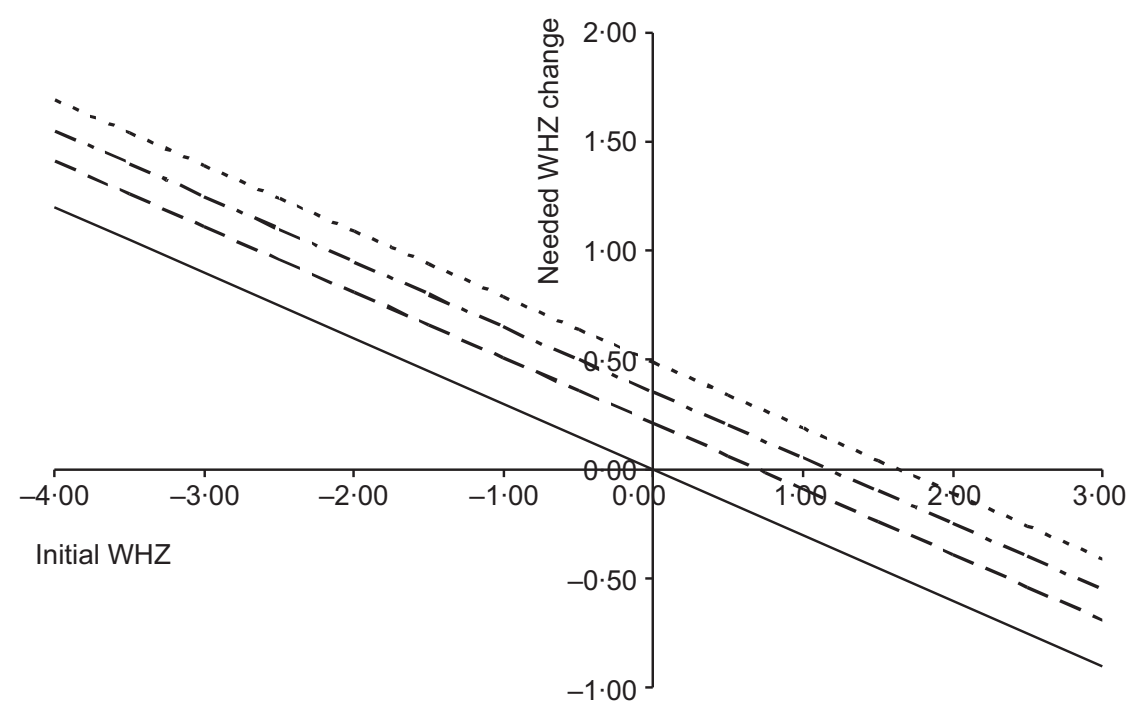

Fig. A1 Change in weight-for-height $Z$-score $(W H Z)$ needed to restore a normal distribution in relation to the initial $W H Z$ for different values of mean initial $\mathrm{WHZ}\left(---\right.$, Mean $\mathrm{WHZ}_{\mathrm{i}}=-0 \cdot 7 ;----$, Mean $\mathrm{WHZ}_{\mathrm{i}}=-0 \cdot 5 ;----, \mathrm{Mean}^{\mathrm{W}} \mathrm{WHZ} \mathrm{Z}_{\mathrm{i}}=-0 \cdot 3$; $\longrightarrow$, Mean $\left.W H Z_{i}=0\right)$. When the mean $W H Z$ in the population is 0 , children in the lower range of $W H Z$ are on average expected to increase $\mathrm{WHZ}$ whereas the children in the upper range are expected to decrease their $\mathrm{WHZ}$, in accordance with the principle of regression to the mean; when the mean $\mathrm{WHZ}$ is negative, children in all $\mathrm{WHZ}$ ranges should have a mean $\mathrm{WHZ}$ change above what is needed when the mean initial $\mathrm{WHZ}$ is 0 


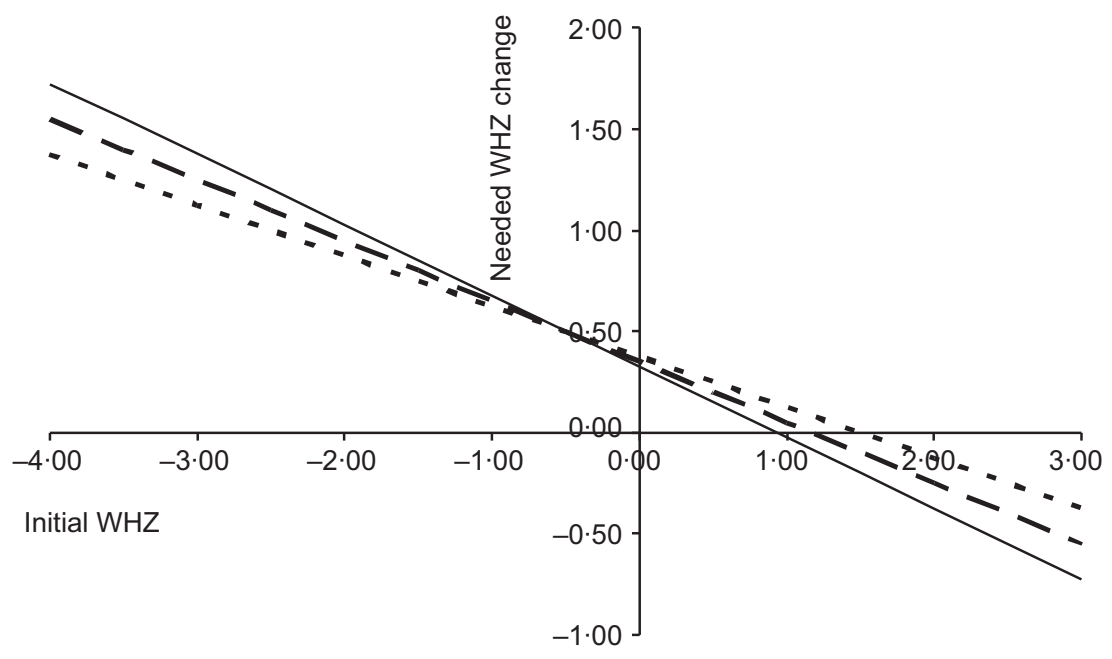

Fig. A2 Change in weight-for-height $Z$-score $(\mathrm{WHZ})$ needed to restore a normal distribution in relation to the initial $W H Z$ for different values of variance of $W H Z$ change $(---, \operatorname{Var} \Delta W H Z=0 \cdot 5 ;----, \operatorname{Var} \Delta W H Z=0 \cdot 6 ;-, \operatorname{Var} \Delta W H Z=0 \cdot 7)$. When variance of $\mathrm{WHZ}$ change increases, the regression to the mean is increased. As a result, $\mathrm{WHZ}$ gain needed for wasted children increases and children with the highest WHZ should decrease their WHZ

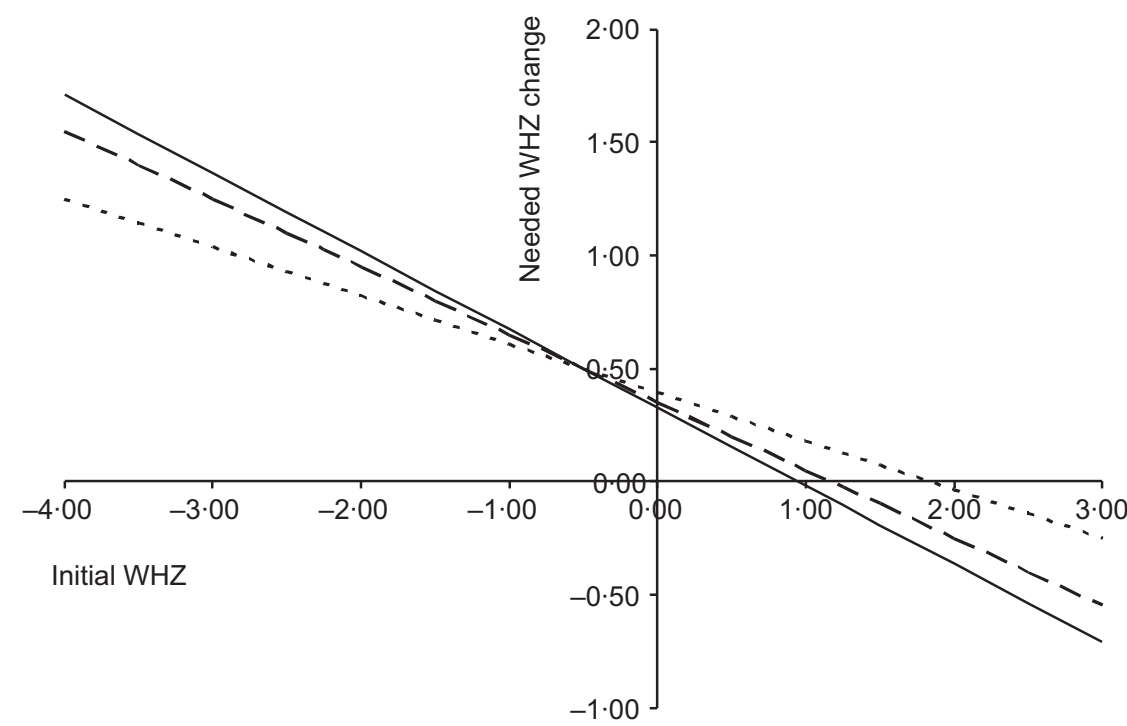

Fig. A3 Change in weight-for-height $Z$-score $(\mathrm{WHZ})$ needed to restore a normal distribution in relation to the initial $W H Z$ for

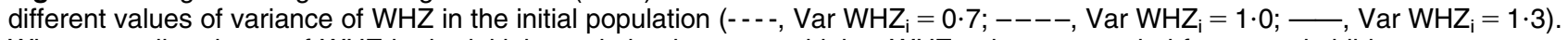
When overall variance of $\mathrm{WHZ}$ in the initial population increases, higher $\mathrm{WHZ}$ gains are needed for wasted children 